\title{
CRISIS INTERVENTION - PROFESSIONAL FIRST AID IN ACUTE CRISIS
}

\section{KRISENINTERVENTION - ERSTE HILFE IN AKUTEN LEBENSKRISEN AUS PROFESSIONELLER SICHT}

\author{
Thomas Kapitany \\ Kriseninterventionszentrum Wien, Wien, Austria
}

\begin{abstract}
SUMMARY
Crisis Intervention is a professional method to help people suffering from acute psychosocial crisis. The basis of effective crisis intervention is a collaborative relationship facilitated by genuineness, respect and acceptance. A thorough assessment of possible endangerment including self harm and suicidal ideations or danger of being a target of violence or even being harmful to others must be established. A facing dialogue offering the possibilities to express and communicate difficult emotional feelings, helping to restore emotional stability and to restore adaptive functioning is an essential basis and might in addition be complemented by medical and/or legal support.
\end{abstract}

Key words: crisis - crisis intervention - suicide - suicidprevention

\section{PSYCHOSOZIALE KRISEN}

Eine psychosoziale Krise oder Lebenskrise wird dadurch definiert, dass eine Person durch ein äußeres Ereignis bzw. äußere Belastungen sein psychisches Gleichgewicht verliert. Dazu kommt es, weil die Belastung aktuell nicht mit den zur Verfügung stehenden Bewältigungsstrategien und Ressourcen verarbeitet werden kann (Sonneck 2000). Auslösend sind traumatische Ereignisse wie Verluste, vitale Bedrohungen aber auch einschneidende Veränderungen im Lebensverlauf.

Lebenskrisen entstehen durch Belastungen im Bereich von Beziehungen (bei Konflikten, Trennungen), im Zusammenhang mit Problemen mit Familie und der Verantwortung für Kindern, bei Verlust durch einen Todesfall, bei der Erkrankung Angehöriger, aber ebenso durch berufliche Entwicklungen und Ereignisse, bei materieller Not oder bei Problemen im Zusammenhang mit Migration und vieles mehr.

Faktoren, die die Entstehung und den Verlauf von psychosozialen Krisen beeinflussen, sind zunächst die Art und Schwere des Krisenanlasses, die subjektive Bedeutung, die ein Krisenanlass für die betroffene Person aufgrund von Vorerfahrungen und aufgrund der aktuellen sozialen Situation hat, die individuelle Krisenanfälligkeit im Rahmen der jeweiligen Persönlichkeitsstruktur, das Fehlen oder Vorhandensein von Ressourcen und Bewältigungsstrategien, und nicht zuletzt die Reaktionen der Umwelt auf das aktuelle Krisengeschehen (siehe Abbildung 1).

\begin{abstract}
ZUSAMMENFASSUNG
Krisenintervention als professionelle Methode stellt ein wertvolles Angebot der Unterstützung von Menschen in einer akuten Lebenskrise dar. Grundlage ist ein unterstützendes, zugewandtes und haltgebendes Beziehungsangebot. Das respektvolle, aktive Ansprechen von selbstdestruktiven Impulsen, Suizidgedanken und-absichten aber auch von anderen sich oder andere gefährdenden Verhaltensweisen ist Grundlage für das Abwenden von gefährlichen Entwicklungen. In vielen Fällen ermöglicht ein entsprechendes Gesprächsangebot bereits eine relevante Entlastung, darüber hinaus können zusätzliche psychosoziale, juristische oder medizinische Maßnahmen notwendig sein.
\end{abstract}

Schlüsselwörter: Psychosoziale Krise - KriseninterventionSuizid - Suizidprävention

Lebenskrisen werden als etwas Normales, zum Lebensverlauf Dazugehöriges angesehen, und nicht als krankheitswertig eingestuft. Bestimmte Lebensphasen oder -übergänge, wie Pubertät, Adoleszenz, oder der Übertritt in den beruflichen Ruhestand weisen eine erhöhte Krisenanfälligkeit auf. Viele Krisen werden alleine oder mit der Hilfe von Angehörigen und Freunden bewältigt.

Abhängig von der Beeinträchtigung der von der Krise betroffenen Person und ihrem Zustand kann die Krisenentwicklung auch krankheitswertig werden. Mögliche Diagnosen des ICD 10, die dann erfüllt sein können, sind bei heftiger Ausprägung aber kurzer Dauer die akute Belastungsstörung F43.0, häufig werden die Kriterien einer Anpassungsstörung F43.2 erfüllt, und bei massiver Traumatisierung durch das auslösende Ereignis mit entsprechender traumaspezifischer Symptomatik ist eine posttraumatische Belastungsstörung F43.1 zu diagnostizieren.

Die Art der Beeinträchtigung und der Symptome, durch die sich die Belastung in einer Krise äußert, kann vielgestaltig sein. Häufig wird ein Gefühl der Überforderung, aber auch der Bedrohung erlebt, das Selbstwertgefühl kann mitunter massiv beeinträchtigt sein. Es bestehen unspezifische Beschwerden, wie Unruhe und Nervosität, Anspannung, Schlaflosigkeit, Sorgen und Gedankenkreisen, Störungen von Konzentration und Aufmerksamkeit. Sehr unterschiedliche Gefühle, wie Trauer, aber auch Ärger oder Wut, Schuld- oder Schamgefühle, - abhängig von der Krisensituation - treten auf. Häufig bestehen Angstgefühle. 


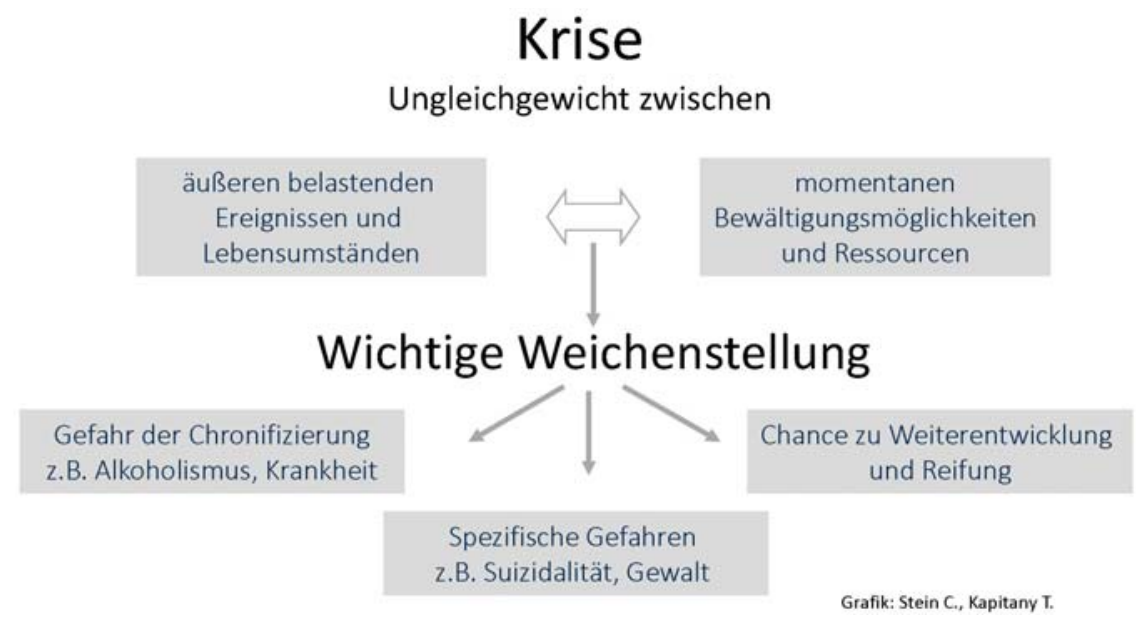

Abbildung 1. Krise

Im Hinblick auf Verlauf und Ausgang einer Lebenskrise wird von einer wichtigen Weichenstellung in der Krise in eine positive oder negative Richtung gesprochen (Stein 2010). Eine positive Entwicklung bedeutet, dass das Krisengeschehen und die Reaktionen darauf gut bearbeitet werden können, notwendige Veränderungen bzw. der Prozess der Problemlösung gelingen und es zu einer Neuorientierung kommt. Die somit positive Bewältigung kann die betroffene Person in ihrem Selbsterleben stärken, zu einer besseren Krisenkompetenz führen und sich positiv auf die Persönlichkeitsentwicklung auswirken (Abbildung 1).

Im Rahmen der Weichenstellung besteht aber auch die erhöhte Gefahr, dass es zu negativen Entwicklungen im Krisengeschehen kommt. Wir müssen dabei spezifische akute Gefährdungen wie massiven symptomatischen Substanzabusus (häufig erhöhter Alkoholkonsum), Suizidalität oder Gewaltgefährdung von der Gefahr chronischer Entwicklungen wie dem Entstehen einer psychischen Erkrankung (z.B. Depression, Alkoholabhängigkeit), einer körperlichen Erkrankung oder der Entwicklung einer prekären sozialen Situation unterscheiden. Damit solche gefährlichen Entwicklungen vermieden bzw. abgefangen werden, kann es entscheidend sein, dass Betroffene rechtzeitig Hilfe durch Krisenintervention von professioneller Seite erhalten (Abbildung 1).

\section{KRISENINTERVENTION}

Professionelle Krisenintervention als Verfahren der Hilfestellung für Menschen in einer akuten psychosozialen Krise hat zum Ziel, zunächst für die betroffene Person eine Entlastung der psychischen Anspannung und des erlebten Drucks zu ermöglichen sowie Gefährdung erkennen und entschärfen zu können. Es sollen Symptome und Beeinträchtigung rasch gelindert werden und dabei die für die Betroffenen notwendige Entscheidungs- und Handlungsfähigkeit wiederhergestellt werden. Damit kann es in weiterer Folge gelingen Lösungen für bestehende Probleme zu erarbeiten.

\section{Prinzipien der Krisenintervention}

Zentrale Prinzipien der Krisenintervention sind eine niederschwellige und rasche Verfügbarkeit der Hilfestellung. Nur so kann das Ziel einer rechtzeitigen und damit in Hinblick auf gefährliche Entwicklungen präventiven Intervention erreicht werden. In der Interaktion mit der betroffenen Person ist ein aktiver und strukturierter Interventionsstil erforderlich, wobei eine Fokussierung auf den Krisenanlass und das Krisengeschehen notwendig ist, damit die Person in der Krise durch eine Reduktion von Komplexität und Überforderung ihre Handlungsfähigkeit wiedererlangen kann. Darüber hinaus ist es notwendig abhängig von den Problemstellungen, dem Zustand der Person in der Krise und der gegenwärtigen sozialen Situation flexibel bezüglich der angewandten Methoden, lösungs- und ressourcenorientiert und im Herangehen bzw. in der weiteren Betreuung und kollegialen $\mathrm{Zu}-$ sammenarbeit multiprofessionell vorzugehen.

\section{Professionelle Helfer}

Kriseninterventionszentren als spezialisierte Institutionen mit einem ambulanten Angebot, einer psychotherapeutischen Fundierung und einer multiprofessionellen Personalausstattung finden sich am ehesten in großen Städten und Ballungszentren. Das spezialisierte persönliche Betreuungsangebot wird in der Regel durch ein telefonisches und zum Teil auch ein Onlineberatungsangebot ergänzt, wodurch das Ziel der größtmöglich niederschwelligen Erreichbarkeit bestmöglich erfüllt wird (z.B. Kriseninterventionszentrum Wien). Ebenso mögliche (erste) professionelle Anlaufstellen für Menschen in einer Lebenskrise sind FachärztInnen für Psychiatrie, PsychotherapeutInnen, Klinische PsychologInnen, SozialarbeiterInnen, aber auch HausärztInnen in eigener Praxis oder BeraterInnen in verschieden psychosozialen Beratungsstellen sowie auch engagierte Laien mit Kriseninterventionsausbildung - häufig im Rahmen von helfenden Telefondiensten bzw. Onlineberatungsanbietern (Telefonseelsorge, Rat auf Draht, etc.) oder im Rahmen von mobilen, aufsuchenden Krisendiensten (Kriseninterventionsteams als Notfalleinsatzkräfte). 


\section{Vorgehen in der Krisenintervention}

Grundlage der Krisenintervention ist ein unterstützendes, der betroffenen Person zugewandtes und haltgebendes Beziehungsangebot und das Herstellen einer solchen therapeutisch wirksamen Beziehung. Maßnahmen, die zum Gelingen dieser Bedingung beitragen, sind die Einbettung des Gesprächs in einen gut vor Störungen geschützten Rahmen und das zur Verfügung stellen von ausreichend Zeit.

Durch die Methode des aktiven Zuhörens vermittelt die HelferIn Interesse an der Problematik und dem psychischen Leid der Person. Durch einen Wechsel von aufmerksamem Zuhören, in dem der Person die Möglichkeit gegeben wird sich mitzuteilen, und aktivem Nachfragen und Ansprechen von auch problematischen, schambesetzten oder tabuisierten Inhalten, kann die oder der Betroffene eine erste Entlastung erleben. Versprachlichung kann dabei helfen, dass eine erste Distanzierung zu belastenden, bedrängenden und auch bedrohlichen Inhalten gelingt.

Durch dieses Herangehen wird der Person das Bemühen und das Wollen vermittelt, sie in ihrem Krisengeschehen zu verstehen, und es kann gelingen, dass sie sich auch mit leidvollen und schwierigen Inhalten akzeptiert und angenommen fühlt, was in der Krisenentwicklung verloren gegangen sein kann oder möglicherweise auch davor nicht ausreichend vorhanden war.

Wenn betroffene Menschen äußern, dass ihr Problem doch nicht bedeutend ist, und es anderen Menschen viel schlechter gehe, ist es hilfreich und wichtig die Krise der Person als solche zu bestätigen. Ein in diesem Sinne psychoedukatives Vorgehen, bei dem Betroffene erfahren, dass die gegebenen Reaktionen und ihr Verhalten im Rahmen eines Krisengeschehens verständlich und üblich sind, hilft eine möglicherweise bereits eingetretene oder drohende Isolation zu reduzieren.

Es ist wichtig für ein hilfreiches Vorgehen in der Krisenintervention, Impulsen vorschnell fertige Lösungen anzubieten widerstehen zu können, auch wenn diese im menschlichen Verhalten verständlich sind. Es ist von großer Bedeutung, dass Helfer in der Krisenintervention aushalten, keine rasche Problemlösung anbieten zu können, und auch darauf verzichten mittels Argumenten von eigenen Einsichten zu überzeugen. Das wichtige haltgebende Erleben akzeptiert $\mathrm{zu}$ werden ist sonst dadurch gefährdet.

\section{Umgang mit Beeinträchtigungen und Gefährdungen}

Weitere wichtige Schritte in der Krisenintervention sind die Befassung mit dem Zustand, den Symptomen und Beeinträchtigungen der Person sowie mit möglichen akuten Gefährdungen. Hier gilt es, sich ein ausreichendes Bild vom Zustand der Person zu machen und Überlegungen anzustellen, wie Symptome und Beeinträchtigung reduziert werden können. Zunächst soll vorrangig versucht werden, bestehende Ressourcen der Betroffenen in der Krise zu aktivieren und zu nützen. Über welche Möglichkeiten und Methoden z.B. der Entspannung oder der natürlichen Schlafförderung verfügt jemand, wie weit lassen sich Personen aus dem Umfeld als Unterstützung heranziehen.

Das Einbeziehen von Anderen ist generell ein wichtiges Mittel in der Krisenintervention. Auch die Helfer sollen nicht um jeden Preis mit ihrer Aufgabe alleine bleiben. Multiprofessionelle Zusammenarbeit z.B. mit ärztlicher Versorgung zur Linderung von Symptomen und Reduktion von Gefährdungen kann eine wichtige Maßnahme darstellen.

\section{Umgang mit Suizidalität}

Ein häufiges Thema der Gefährdung bei Menschen in einer akuten Lebenskrise ist das Entstehen einer Suizidgefährdung. Indem durch das Erleben von Verlust und Überforderung wesentliche persönliche Werte und Erwartungen in Frage gestellt werden, der Selbstwert erheblich beeinträchtigt ist und schwer aushaltbare Gefühle und Zustände entstehen, passiert es nicht selten, dass Betroffene zunehmend verzweifelt ihr Leben in seiner Sinnhaftigkeit in Frage stellen. Oder es wird das eigene Leben als nicht mehr aushaltbar angesehen. Aus diesen Gründen denken Betroffene dann daran ihr Leben beenden zu wollen. 30\% der Personen, die in einer psychosozialen Krise zur persönlichen Betreuung in das Kriseninterventionszentrum Wien kommen, haben Suizidgedanken, bei $20 \%$ bestehen ernsthafte bzw. konkrete Suizidvorstellungen.

In der Krisenintervention ist es daher entscheidend sich im Gespräch offen und vorbehaltlos dem Thema Suizidalität zuzuwenden. Im Vorgehen muss zunächst daran gedacht werden, dass eine Suizidgefährdung gegeben sein kann, um dann aktiv dieses Thema anzusprechen und nachzufragen, ob Suizidgedanken und absichten bestehen. So ist bei jemandem, der sich in einer Krise befindet, die mögliche Zugehörigkeit zu einer Risikogruppe (z.B. Menschen mit einer psychischen Erkrankung; Männer, besonders im höheren Alter; Suizide in der Familie; frühere Suizidversuche) und das Vorhandensein von Risikofaktoren (erleichterter Zugang zu Suizidmitteln, wie z.B. Schusswaffen oder Medikamente; Identifikations-/Imitationseffekte) bei der Einschätzung der Suizidgefährdung zu berücksichtigen.

Schließlich soll vorbehaltlos und wertfrei mit der betroffenen Person über Suizidgedanken gesprochen werden. So kann sie Ängste und Schamgefühle im Zusammenhang mit dem schwierigen Thema der Suizidalität reduzieren und sich aussprechen, wodurch wiederum eine erste Entlastung eintreten kann und die helfende Beziehung weiter gefestigt wird.

In der Einschätzung der Suizidalität geht es im nächsten Schritt darum abzuklären, in welcher Form und in welchem Ausmaß diese Gedanken vorhanden sind, ob konkrete Vorstellungen oder gar Handlungspläne bestehen, bzw. ob bereits Vorbereitungen getroffen wurden. Je konkreter die Gedanken sind, je häufiger und drängender sie auftreten, desto erheblicher ist die Gefährdung (Abbildung 2). 


\section{Einschätzung der Suizidgefährdung}

\begin{tabular}{|c|c|c|c|c|}
\hline Basales Suizidrisiko & \multicolumn{4}{|c|}{ Aktuelle Suizidalität } \\
\hline $\begin{array}{l}\text { Risikogruppe/ } \\
\text { Risikofaktoren }\end{array}$ & $\begin{array}{c}\text { Suizidale } \\
\text { Inhalte/ } \\
\text { Entwicklung }\end{array}$ & $\begin{array}{c}\text { Krise/ } \\
\text { Trauma/ } \\
\text { Soziale } \\
\text { Situation }\end{array}$ & $\begin{array}{c}\text { Präsuizidales } \\
\text { Syndrom/ } \\
\text { Affekte }\end{array}$ & $\begin{array}{l}\text { Beziehungs- } \\
\text { gestaltung }\end{array}$ \\
\hline $\begin{array}{c}* \text { Psychische Erkrankung } \\
\text { Ältere Menschen (bes. } \\
\text { Männer) } \\
* \text { Suizidversuch in der } \\
\text { Vorgeschichte } \\
* \text { Leichter Zugriff auf } \\
\text { tödliche Mittel } \\
* \text { Suizide in der Familie } \\
* \text { Imitationseffekt }\end{array}$ & $\begin{array}{l}\text { Gedanken } \\
\text { Ankündigung } \\
\text { Hinweis(e) } \\
\text { Erwägung } \\
\text { Abwägung } \\
\text { Entschluss }\end{array}$ & $\begin{array}{c}\text { Akute äußere } \\
\text { Belastung } \\
\text { Ressourcen, } \\
\text { persönliche Bedeutung } \\
\text { Soziale Isolation }\end{array}$ & $\begin{array}{c}\text { Hoffnungslosigkeit } \\
\text { Angst } \\
\text { Verzweiflung }\end{array}$ & $\begin{array}{l}\text { Unerreichbarkeit } \\
\text { (Anzeichen für) } \\
\text { Dissimulation } \\
\text { Mangelnde } \\
\text { Kooperations- } \\
\text { bereitschaft oder } \\
\text { - fähigkeit }\end{array}$ \\
\hline
\end{tabular}

Abbildung 2. Einschätzung der Suizidgefährdung (C. Stein, T. Kapitany)

In weiterer Folge ist zu beurteilen, ob die/der Betroffene noch positiv affiziert werden kann, d.h. zu einer positiven emotionalen Reaktion auf entsprechende Inhalte fähig ist, oder ob die Person in ihrem Zustand erheblich affektiv und gedanklich inhaltlich eingeengt ist, wie Ringel es in seinem präsuizidalen Syndrom beschreibt (1956), was als weiteres Indiz für ein Suizidrisiko gewertet werden muss (Abbildung 2).

Mit dem Grad der Einengung unter Umständen übereinstimmend ist die Art der Beziehungsgestaltung der Person in der Krise zu beurteilen. Es gilt einzuschätzen, ob die/der Betroffene soweit im Gespräch auf der Ebene des Kontakts erreicht werden kann, dass eine verlässliche Vereinbarung für den Zeitraum bis zu einem nächsten Behandlungs-/Gesprächstermin getroffen werden kann, bei der ausreichende Sicherheit für die Person gewährleistet ist (Abbildung 2). Teil einer solchen Vereinbarung müssen auch Notfallmaßnahmen (verfügbare hilfreiche Angehörige; telefonische, ambulante und mobile Notfalldienste; Bedarfsmedikation) für mögliche akute Zuspitzungen sein, damit in jedem Fall rechtzeitig Hilfe geholt werden kann. Wenn mangelnde Kooperationsbereitschaft bzw. Kooperationsfähigkeit festgestellt wird, müssen bei entsprechenden suizidalen Inhalten unmittelbar durch die Helfer Notfallmaßnahmen eingeleitet werden (Transfer zu einem Notfalldienst, in eine psychiatrische Krankenhausabteilung; Rettung und/oder Polizei verständigen; etc.).

\section{Begleitung während der Zeit der Krise}

Während es in einer Krise auch bei einer einmaligen Intervention bzw. Beratung von professioneller Seite bleiben kann, wird das Angebot der Krisenintervention meist während des Zeitraums der Krise in bis zu 10 Gesprächsterminen genutzt. Ziel ist, zunehmend an den Lösungen der Probleme und an einer beginnenden Neuorientierung arbeiten zu können. Wichtiges Element der Krisenintervention ist auch eine gute Beendigung des Prozesses, mit dem Ziel die Erfahrungen mit einer Krise und ihren Auslösern und das Erleben der Bewältigung gut in das eigene Leben zu integrieren und somit auch für zukünftige krisenhafte Situationen nützen zu können.

Acknowledgements: None.

Conflict of interest: None to declare.

\section{Literatur}

1. Kriseninterventionszentrum Wien: Tätigkeitsbericht 2018. Eigenverlag 2019.

Online: www.kriseninterventionszentrum.at/wpcontent/uploads/2019/09/Tätigkeitsbericht_2018_kv.pdf

2. Sonneck G: Krisenintervention und Suizidverhütung. Facultas Universitätsverlag, 2000

3. Stein C: Spannungsfelder der Krisenintervention, Kohlhammerverlag, 2009

\section{Correspondence:}

Thomas Kapitany, MD

Kriseninterventionszentrum Wien

Wien, Austria

E-mail: thomas.kapitany@neubaugasse3.at 